\title{
Zur Ikonizität deutscher Zwillingsformeln
}

\author{
Agnieszka Gaweł (Krakau)
}

\begin{abstract}
In this paper we present a corpus analysis of the influence of sequential iconicity on the linear order of German irreversible binomials. The analysis provides evidence for the thesis that semantic and pragmatic factors determining the linear order of binomials can be interpreted as examples of sequential iconicity which subsumes several cases of iconic coding: the linear order of linguistic units reflecting the structure of the extralinguistic reality, the cognitive processes and the sociocultural coding.
\end{abstract}

\section{$1 \quad$ Ikonizität und Ikonizitätsforschung}

Der Terminus Ikon wird in den semiotischen Untersuchungen von Charles Peirce zur Bezeichnung von Zeichen eingeführt, welche zu ihrem Repräsentamen Ähnlichkeit aufweisen, im Unterschied zu Indizes, die in keiner Similaritätsbeziehung zu den von ihnen vertretenen Referenzobjekten stehen, bei denen jedoch eine natürliche Verbindung zwischen dem Zeichen und dem Referenzobjekt anderer Art als formale Ähnlichkeit besteht, und Symbolen, deren Beziehung zum Referenzobjekt ausschließlich auf Konvention beruht (cf. Peirce 1903/1998: 273). Der Terminus Ikonizität wird zum ersten Mal von Charles Morris zur Bezeichnung des Ähnlichkeitsgrades zwischen einem ikonischen Zeichen und seinem Referenzobjekt verwendet. Morris weist nämlich darauf hin, dass sich die einzelnen Ikons dadurch voneinander unterscheiden, in welchem Grade sie zu den von ihnen bezeichneten Objekten Ähnlichkeit aufweisen (cf. Morris 1938/1964: 68).

Die von Peirce und Morris initiierten Untersuchungen zur Charakteristik der zeichenkonstitutiven Beschaffenheit der Ikons beeinflussen die späteren Forschungen zur Ikonizität in der Sprache, die im Rahmen verschiedener linguistischer Strömungen (insbesondere der Kognitiven Linguistik und der funktionalistischen Typologieforschung) vorgenommen werden. Sie eröffnen nämlich die für die Ikonizitätsforschung grundlegende Diskussion über die Art der Beziehung zwischen dem ikonischen Zeichen, dem Interpretanten und dem Referenzobjekt. Zentral in dieser Diskussion ist die Frage, inwieweit die ikonischen Zeichen ausschließlich in Relation zu den ihnen entsprechenden Referenzobjekten interpretiert werden können, und inwieweit in der Ikonizitätsforschung die Einbeziehung des Konzeptualisierers notwendig ist, der sowohl das Zeichen als auch die Wirklichkeit auf bestimmte Art und Weise wahrnimmt. Diesbezüglich unterscheiden sich die linguistischen Zugänge zur Ikonizität dadurch, welche Rolle im jeweiligen Ansatz den konzeptuellen Prozessen zugeschrieben wird, die mit der Wahrnehmung, Konstruktion und Strukturierung der Wirklichkeit verbunden sind. Die erste

Linguistik online 81, 2/17 - http://dx.doi.org/10.13092/lo.81.3645

CC by 3.0 
Gruppe bilden Untersuchungen, die von der objektivierten Definition der Ikonizität ausgehen und die Ikons als Zeichen definieren, welche Ähnlichkeit zu ihren Referenzobjekten aufweisen (cf. Wescott 1971: 416). Die zweite Gruppe bilden Untersuchungen, die auf die Rolle der Wahrnehmung bei der Produktion und Rezeption ikonischer Zeichen verweisen. In diesen Ansätzen wird betont, dass die angesprochene Similaritätsbeziehung nicht unmittelbar Ikons und ihre Referenzobjekte betrifft, sondern Ikons und die wahrgenommenen Referenzobjekte (cf. Dotter 1995: 48). Hierzu gehören auch Untersuchungen, in denen die Rolle der Konzeptualisierung bei der Produktion und Rezeption ikonischer Zeichen besonders stark hervorgehoben wird und welche die Ikonizität als Abbild der Struktur der Erfahrung bzw. der individuellen und oft subjektiven Betrachtungsweise der Welt durch den Konzeptualisierer definieren (cf. Croft 1995: 129; Tabakowska 2005: 376). Die dritte Gruppe bilden Untersuchungen, in denen neben dem Zusammenhang zwischen sprachlicher Ikonizität und kognitiven Prozessen der Zusammenhang zwischen Ikonizität und anderen Modi sprachlicher Kodierung, insbesondere der kulturellen Kodierung, betont wird (cf. Givón 1989: 74).

\section{Zur sequentiellen Ikonizität in der Syntax}

In der linguistischen Forschung wurden zahlreiche Klassifizierungsvorschläge der Ikons bearbeitet, die für die Untersuchungen zur sequentiellen Ikonizität in der Syntax von Relevanz sind (cf. z. B. Haiman 1985: 11; Givón 1995: 49-56; Andersen 1998: 265-313; Haspelmath 2003: 2). Da die Besprechung aller erwähnten Ansätze den Rahmen des vorliegenden Beitrags sprengen würde, beschränken wir uns hier auf die Darstellung des Klassifizierungsvorschlags von Givón (1995) und der von Andersen (1998) durchgeführten Untersuchungen zur sequentiellen Ikonizität in der Syntax.

Givón (1995: 49-56) unterscheidet drei Prinzipien, die mit der ikonischen Kodierung in der Syntax verbunden sind: das Quantitäts-, das Abstands- und das Abfolgeprinzip. Das Quantitätsprinzip bezieht sich auf die Tatsache, dass typischerweise die Menge vom sprachlichen Material mit der Anzahl, der Unvorhersehbarkeit bzw. der Relevanz der zu vermittelnden Informationen korrespondiert. Das Abstandsprinzip kommt in syntaktischen Strukturen zum Ausdruck, in denen der sprachliche Abstand den funktionalen, konzeptuellen bzw. kognitiven Abstand abbildet. Das Abfolgeprinzip, das für die hier vorgenommenen Untersuchungen von besonderer Relevanz ist, umfasst zwei Subprinzipien: das semantische und das pragmatische Abfolgeprinzip. Das semantische Abfolgeprinzip verweist darauf, dass die lineare Abfolge sprachlicher Einheiten mit der zeitlichen Abfolge von Ereignissen in der außersprachlichen Wirklichkeit korrespondiert. Ein Beispiel für die Anwendung des genannten Prinzips ist die ikonische Abbildung der zeitlichen Abfolge von Ereignissen in Satzverbindungen mit der Konjunktion ,und':

Sie stand auf und machte sich an die Arbeit.

Das pragmatische Abfolgeprinzip verweist auf zwei Tendenzen: die Tatsache, dass wichtigere Informationen bzw. Informationen, die dringend vermittelt werden sollen, in der Regel am Anfang des Satzes bzw. am Anfang der Phrase platziert werden, sowie auf die Tatsache, dass Informationen, die schwerer zugänglich bzw. weniger vorhersehbar sind, in der Regel am 
Anfang des Satzes platziert werden. ${ }^{1}$ Ein Beispiel für die Anwendung des genannten Prinzips ist die Platzierung der Interrogativpronomina und -adverbien am Anfang des Fragesatzes:

Wen hast du gesehen?

Andersen (1998: 267-288) unterscheidet zahlreiche semantische Faktoren, die durch die Reihenfolge sprachlicher Einheiten ikonisch abgebildet werden. Hierzu gehören:

- die zeitliche Abfolge von Ereignissen (Präferenz zur Voranstellung der sprachlichen Einheit, die auf zeitlich vorangehende Ereignisse Bezug nimmt, z. B. heute oder morgen)

- die Hierarchie (Präferenz für die Voranstellung der sprachlichen Einheit, die auf die höhere Position in der Hierarchie verweist, z. B. Vater und Sohn)

- Präferenz (Tendenz zur Voranstellung der Einheit, die auf bevorzugte Gegenstände, Personen bzw. Sachverhalte Bezug nimmt, z. B. Gold und Silber)

- Anfang, Mitte und Ende (Präferenz für die Beibehaltung der linearen Abfolge Anfang Ende, Anfang - Mitte - Ende, z. B. Anfang und Ende $)^{2}$

- räumliche Sequenzen (die ikonische Abbildung der Bewegung in eine bestimmte Richtung, z. B. am Barbakan vorbei, über den unterirdischen Übergang bis zum Bahnhof)

- progressive Serien (die ikonische Abbildung der Progression in Anzahl, Länge bzw. Größe, z. B. eins, zwei, drei).

\section{Bisherige Untersuchungen zur linearen Abfolge von Bestandteilen der Zwillings- formeln}

Mit dem Terminus Zwillingsformel bezeichnen wir im vorliegenden Text in Anlehnung an Burger (2010: 45) eine formelhafte Verbindung von zwei verschiedenen Wörtern, die der gleichen Wortart angehören und mit einer Konjunktion bzw. Präposition zu einer phraseologischen Einheit verbunden werden (dabei weist die Reihenfolge von Bestandteilen der entstandenen phraseologischen Einheit wesentliche Merkmale der Festigkeit auf) bzw. eine feste Verbindung, in der zwei gleiche Wörter mit einer Konjunktion oder Präposition zu einer phraseologischen Einheit verbunden werden. ${ }^{3}$ Da die Reihenfolge von Bestandteilen einer Zwillingsformel entweder irreversibel ist oder zumindest eine starke Präferenz für die Verwendung einer Reihenfolge beobachtet werden kann, stellt sich die Frage, ob und welche Regeln bzw. Tendenzen die Reihenfolgebeziehungen in Zwillingsformeln determinieren. In den bis-

\footnotetext{
${ }^{1}$ Givóns Vorschlag ist insofern umstritten, als aus pragmatischer Sicht nicht ausschließlich die oben erwähnte Tendenz zur Platzierung wichtigerer und schwerer zugänglicher bzw. weniger vorhersehbarer Informationen am Anfang des Satzes als ikonisches Abbild des Kommunikationsprozesses betrachtet werden kann, sondern auch die sprachenübergreifend zu beobachtende Präferenz zur Voranstellung des Themas, die mit der Tendenz zur Anknüpfung an bereits bekannte Informationen in Verbindung steht.

2 Andersen (1998: 284-285) vertritt in seinen Untersuchungen zur Hierarchie Anfang - Mitte - Ende sogar die radikalere These, dass in vielen literarischen Werken sprachliche Einheiten, die auf das Ende Bezug nehmen, häufiger als Einheiten, die auf den Anfang bzw. die Mitte Bezug nehmen, am Ende des Satzes aufträten etc.

${ }^{3}$ Die hier angebrachte Definition, so wie sie bei der Selektion des Materials für die Korpusanalyse interpretiert wird, schließt nicht die Möglichkeit aus, dass in einer Zwillingsformel neben ihren Hauptbestandteilen Negationswörter auftreten bzw. Elemente, die der Kennzeichnung grammatischer Kategorien dienen.
} 
herigen Untersuchungen zu Zwillingsformeln wurden vier Gruppen von Faktoren identifiziert, die einen Einfluss auf die lineare Abfolge von Bestandteilen vieler Zwillingsformeln haben:

1. Regeln und Tendenzen, die mit der formalen Charakteristik der einzelnen Bestandteile einer Zwillingsformel verbunden sind (cf. Malkiel 1959: 149-151; Ross 1980: 40-48; Müller 1997: 18-35; Müller 2009: 49-89; Jarosz 2009: 53-54).

Hierzu gehören solche Faktoren wie Vokalkontrast, Größe des Silbenvorlaufs, Größe vom Nukleus der Silbe, die Sonoritätshierarchie der Konsonanten, Silbenzahl und die maximale rhythmische Alternation. ${ }^{4}$

2. Regeln und Tendenzen, die mit der Entstehung neuer Zwillingsformeln im Zusammenhang stehen (cf. Malkiel 1959: 151-154; Schröter 1980: 194; Hüpper/Topalović/Elspaß 2002: 8596).

Die Reihenfolge von Bestandteilen vieler Zwillingsformeln kann ausschließlich im Kontext der Verfestigungsprozesse erklärt werden, die mit dem Sprachgebrauch im Zusammenhang stehen (cf. Hüpper/Topalović/Elspaß 2002: 85-96). Darüber hinaus ergibt sich die Unumkehrbarkeit mancher Zwillingsformeln daraus, dass die jeweilige phraseologische Einheit als Analogiebildung zu einer anderen populären Zwillingsformel in derselben Sprache bzw. als Lehnübersetzung einer Zwillingsformel in einer Fremdsprache entstanden ist oder infolge der Rezeption eines literarischen Werkes bzw. Liedes popularisiert wurde (cf. Malkiel 1959: 151154; Schröter 1980: 194; Benor/Levy 2006: 268-269).

3. Regeln und Tendenzen, die mit der semantischen bzw. pragmatischen Charakteristik der einzelnen Bestandteile einer Zwillingsformel verbunden sind (cf. Malkiel 1959: 143-149; Cooper/Ross 1975: 65-67; Müller 1997: 15-18; Müller 2009: 80-87; Jarosz 2009: 51-53).

In diesem Kontext verweist die Fachliteratur auf das Vorhandensein semantischer und pragmatischer Hierarchien, welche die lineare Abfolge von Bestandteilen der Zwillingsformeln determinieren. Müller (2009: 80-87) unterscheidet in seinen Untersuchungen zu Reihenfolgebeziehungen in Zwillingsformeln die folgenden semantischen und pragmatischen Hierarchien:

- Belebt - unbelebt

- Männlich - weiblich

- Menschlich - nicht menschlich

- Erwachsen - nicht erwachsen

- Für den Menschen wichtigere Tiere - unwichtigere Tiere

- Höherstehende - Tieferstehende / Untergebene

- $\quad$ Nah - fern

- Zeitliche Abfolge

\footnotetext{
${ }^{4}$ Eine weitere metrische Beschränkung, deren Einfluss auf die Reihenfolge von Bestandteilen englischer Wortpaare bestätigt wurde, ist die Tendenz zur Vermeidung der Endsilbenbetonung (cf. Bolinger 1962). Da jedoch die Relevanz der genannten Tendenz für die Beschreibung deutscher Zwillingsformen bisher nicht erforscht wurde, wird dieser Faktor in der Auflistung oben nicht berücksichtigt.
} 
- Unmarkiertes vor Markiertem

- $\quad$ Allgemein - speziell

- Nahrungshierarchie

- Mehr vor weniger Alkohol

- Menschliches - Funktion / Eigentum

- Positiv - negativ

- Wertvoll - weniger wertvoll

- Moralisch weniger Verwerfliches - moralisch Verwerflicheres

- Wichtige Körperteile - weniger wichtige Körperteile

- Mehr Quantität - weniger Quantität

- $\quad$ Oben - unten

- $\quad$ Tier - Gegenstand/Ort

- Konkretes mit Echo

4. Regeln und Tendenzen, die sich aus der Vorkommensfrequenz von Bestandteilen der Zwillingsformeln ergeben (cf. Fenk-Oczlon 1989: 517-537).

Das Wort mit der höheren Vorkommensfrequenz geht in der Regel dem Wort mit der niedrigeren voran.

Darüber hinaus sind in der bisherigen Forschung zu Reihenfolgebeziehungen in Zwillingsformeln zahlreiche Versuche vorgenommen worden, die einzelnen semantischen und phonologischen Beschränkungen auf ihnen übergeordnete allgemeinere Prinzipien zurückzuführen. Cooper/Ross (1975: 67) und Ross (1980: 48) vertreten die These, dass die meisten der bei Cooper/Ross (1975) vorgeschlagenen semantischen Regeln mit der Ich-vor-allem-Regel in Verbindung gebracht werden können. Im Einklang mit der Ich-vor-allem-Regel verweist das Erstglied einer Zwillingsformel auf die Merkmale eines prototypischen Sprechers, der u. a. solche Eigenschaften wie ,hier und jetzt anwesend', ,männlich', ,erwachsen', ,positiv ‘ u. ̈̈. in sich vereint. Cooper/Ross (1975: 67) betonen jedoch die Tatsache, dass nicht alle der in ihren Untersuchungen identifizierten semantischen Hierarchien auf die Ich-vor-allem-Regel zurückgeführt werden können. Ausnahmen bilden die Hierarchie göttlich - weltlich und die von ihnen vorgeschlagene Hierarchie Pflanze - Tier ${ }^{5}$. Szpyra (1983) vertritt die Ansicht, dass die Ich-vor-allem-Regel potentiell eine Erklärung für weitere, in der Generalisierung von Cooper/Ross unberücksichtigte semantische Hierarchien liefern kann, wenn sie um eine zusätzliche Komponente erweitert wird. Nach Szpyra (1983: 42) spiegeln nämlich die Reihenfolgebeziehungen in Zwillingsformeln nicht ausschließlich prototypische Eigenschaften des Sprechers wider, sondern auch die Hierarchie der Werte, die für die Gesellschaft, in der er lebt, charakteristisch ist. Bei Müller (1997: 15) werden die semantischen Hierarchien, welche

\footnotetext{
${ }^{5}$ Die Relevanz der zweitgenannten Hierarchie für die Beschreibung der Reihenfolgebeziehungen in Zwillingsformeln wurde durch spätere Untersuchungen nicht bestätigt.
} 
die Reihenfolge von Bestandteilen der Zwillingsformeln determinieren, genauso wie bei Szpyra (1983), mit gesellschaftlichen Werten in Verbindung gebracht, die z. T. kulturspezifisch sind. Aus diesem Grund wird bei Müller (1997) zur Bezeichnung semantischer Hierarchien der Terminus Salienzbeschränkungen verwendet, der signalisiert, dass in Zwillingsformeln typischerweise das in der jeweiligen Sprachgemeinschaft höher Geschätzte bzw. Wichtigere dem geringer Geschätzten bzw. weniger Wichtigen vorangeht. Auch in den Untersuchungen zum Einfluss phonologischer Beschränkungen auf die Reihenfolgebeziehungen in Zwillingsformeln spielt die Suche nach allgemeinen Prinzipien, auf welche die einzelnen phonologischen Regeln zurückgeführt werden können, eine wichtige Rolle. Cooper/Ross (1975: 80) unternehmen den Versuch, die von ihnen formulierten phonologischen Beschränkungen mit der Tendenz zur Reduzierung des Erstgliedes einer Zwillingsformel in Verbindung zu bringen. Sie weisen jedoch darauf hin, dass nur gewisse phonologische Beschränkungen als Ausdruck der genannten Tendenz betrachtet werden können. Ausnahmen bilden z. B. die Vokalhierarchie und die Sonoritätshierarchie der Konsonanten.

Neben Prinzipien, die sich ausschließlich auf semantische bzw. phonologische Faktoren beziehen, wird bei Ross (1980) und Sobkowiak (1993) auf die Existenz noch allgemeinerer Tendenzen hingewiesen, die sowohl semantische als auch phonologische Beschränkungen für die Anordnung von Bestandteilen der Zwillingsformeln umfassen. Ross (1980: 48) formuliert die These, dass in Zwillingsformeln die semantische Unmarkiertheit mit phonetischer Unhörbarkeit korrespondiere. Die phonologische Charakteristik des prototypischen Erstgliedes einer Zwillingsformel könne folglich als ein ikonisches Abbild ihrer semantischen Eigenschaften betrachtet werden. Sobkowiak (1993: 395) vertritt die Ansicht, dass die einzelnen Regeln, welche die Reihenfolge von Bestandteilen der Zwillingsformeln determinieren, auf ein einziges semantisch-phonologisches Prinzip zurückgeführt werden können: Das unmarkierte Glied einer Zwillingsformel gehe in der Regel dem markierten Glied voran. ${ }^{6}$

\section{Bisherige Untersuchungen zur Ikonizität der Zwillingsformeln und Wortpaare}

In der bisherigen Forschung zur Ikonizität in der Phraseologie (cf. z. B. Ross 1980; Munat 2005; Farø 2006a, 2006b; Römer 2007) wurde der Relevanz von Ikonizitätsprinzipien für die Beschreibung der Zwillingsformeln nur wenig Aufmerksamkeit geschenkt. Die Anzahl der uns bekannten Veröffentlichungen, in denen die Ikonizität der Zwillingsformeln den primären Untersuchungsgegenstand darstellt, beschränkt sich auf drei Beiträge: Ross (1980); Kimenyi (1989) und Southern (2000).

In den bereits präsentierten Untersuchungen von Ross (1980) liegt der Analyseschwerpunkt auf der Frage, inwieweit die einzelnen semantischen und phonologischen Beschränkungen für die lineare Anordnung von Bestandteilen der Zwillingsformeln auf ihnen übergeordnete allgemeinere Prinzipien zurückgeführt werden können. Er kommt dabei zum Schluss, dass anhand der bei Cooper/Ross (1975) und Ross (1980) identifizierten Beschränkungen prototypische Merkmale des Erstgliedes einer Zwillingsformel rekonstruiert werden können, in dem

\footnotetext{
${ }^{6}$ Ross (1980) verwendet den Terminus semantische Unmarkiertheit undefiniert zur Bezeichnung von Elementen, die auf Eigenschaften eines prototypischen Sprechers Bezug nehmen. Der bei Sobkowiak (1993) verwendete Terminus markedness (dt. Markiertheit) bezieht sich allgemein auf den Kontrast zwischen einer merkmaltragenden und merkmallosen Einheit, die miteinander korreliert sind (cf. Sobkowiak 1993: 396).
} 
die semantische Unmarkiertheit mit phonetischer Unhörbarkeit korrespondiere (cf. Ross 1980: 48).

Den Untersuchungsgegenstand bei Kimenyi (1989) bildet die syntagmatische Lautsymbolik in englischen Zwillingsformeln. Die Ergebnisse seiner Analyse indizieren, dass im Englischen eine Untergruppe von Zwillingsformeln identifiziert werden kann, in denen die phonetische Ähnlichkeit zwischen ihren Bestandteilen (die beiden Bestandteile teilen mindestens einen Laut) mit der semantischen Similaritätsbeziehung zwischen diesen Bestandteilen korrespondiert (die beiden Bestandteile weisen wesentliche Ähnlichkeiten in ihrer semantischen Charakteristik auf) (cf. Kimenyi 1989: 350-352). ${ }^{7}$

Southern (2000) analysiert die Relevanz quantitativer Ikonizität für die Beschreibung von Beziehungen zwischen Form und Bedeutung der Zwillingsformeln in westgermanischen Sprachen. Im Mittelpunkt seines Interesses liegt die Ikonizität von Zwillingsformeln, deren Bestandteile durch Stab- bzw. Endreim miteinander verbunden sind, seine theoretischen Überlegungen sind aber auch für andere Typen von Zwillingsformeln relevant, deren Bestandteile bzw. prosodische Einheiten Ähnlichkeiten in ihrem Aufbau aufweisen. Er kommt dabei zum Schluss, dass für viele Zwillingsformeln in westgermanischen Sprachen die sog. prosodische Komplementarität (engl. prosodic complementarity) charakteristisch sei, welche in folgenden Formen auftreten könne (cf. Southern 2000: 256, 258):

- $\quad$ Stabreim (z. B. klipp und klar)

- $\quad$ Endreim (z. B. rütteln und schütteln)

- Symmetrie der Silbenstruktur (z. B. laufen und rennen)

- $\quad$ Sprachinterne rhythmische Euphonie der Sequenz ${ }^{8}$ (z. B. Leib und Seele).

Gleichzeitig weisen Zwillingsformeln aus den genannten Gruppen Ähnlichkeiten in ihrer semantischen Charakteristik auf, da die Gesamtbedeutung ihrer Komponenten in der Regel Totalität ausdrückt. Zum Ausdruck der Totalität werden in westgermanischen Zwillingsformeln verschiedene Strategien verwendet (cf. Southern 2000: 261):

- $\quad \mathrm{p}+-\mathrm{p}=$ das Gesamte (Antonymie, z. B. Himmel und Hölle)

- $\quad \mathrm{p}+\Delta \mathrm{p}=$ das Gesamte (Synonymie, z. B. verriegelt und verschlossen)

- $\quad \mathrm{p}+\mathrm{q}=$ das Gesamte (Merismus, Teil-Ganzes-Beziehung, z. B. Kind und Kegel).

\footnotetext{
${ }^{7}$ Die Relevanz der bei Kimneyi (1989) formulierten Thesen für die Beschreibung deutscher Zwillingsformeln wird durch die Untersuchungsergebnisse von Southern (2000) bestätigt. Darüber hinaus sind für die Beschreibung der quantitativen Ikonizität in deutschen Zwillingsformeln die Untersuchungen von Mellado Blanco (1998) von besonderer Relevanz, die zwar keine expliziten Bezüge auf die quantitative Ikonizität enthalten, in denen jedoch der Zusammenhang zwischen dem expressiv verstärkenden Charakter vieler somatischer Zwillingsformeln und den formalen Eigenschaften der genannten Phraseologismen (die zweigliedrige Struktur mit der Konjunktion und, Stabreim, Endreim und Assonanz) analysiert wird (cf. Mellado Blanco 1998: 289-290).

${ }^{8}$ Der Terminus sprachinterne rhythmische Euphonie der Sequenz (engl. language-internal rhythmic euphony of sequence) bezieht sich in diesem Kontext auf die prosodische Komplementarität in Zwillingsformeln, deren Bestandteile sich in ihrer Länge ausschließlich um eine Silbe unterscheiden und in denen die Beibehaltung des bei Behaghel $(1909,1932)$ formulierten Gesetzes der wachsenden Glieder dazu beiträgt, dass das Erstglied samt der ihm folgenden einsilbigen Konjunktion die gleiche Silbenstruktur wie das Zweitglied hat.
} 
Die prosodische Komplementarität in den genannten Gruppen von Zwillingsformeln diene folglich nach Southern (2000: 260) der ikonischen Abbildung semantischer Beziehungen zwischen ihren Bestandteilen.

Darüber hinaus finden sich in der Fachliteratur zu Zwillingsformeln und zur Ikonizität in der Sprache vereinzelte Bezüge auf bestimmte auf Prinzipien der Ikonizität zurückzuführende Eigenschaften der genannten Gruppe von Phraseologismen. So verweist z. B. Andersen (1998: 267-268) auf die Tatsache, dass die von ihm identifizierten semantischen Faktoren, welche durch die lineare Abfolge sprachlicher Einheiten ikonisch abgebildet werden, auch die Reihenfolgebeziehungen in Zwillingsformeln determinieren. Plank (1979: 140-141) betont die Relevanz perzeptueller Markiertheit für die Beschreibung von Zwillingsformeln, deren Bestandteile sich auf die Position auf der vertikalen Achse beziehen. Er formuliert dabei die These, dass die zu beobachtende Tendenz für die Voranstellung von Elementen, die auf oben gelegene Entitäten verweisen, den prototypischen Fall der visuellen Wahrnehmung von oben nach unten ikonisch abbildet. Plank (1979: 141) und Mellado Blanco (1998: 292) vertreten die Ansicht, dass auch die Abweichung von der aus der Sicht der perzeptuellen Markiertheit bevorzugten Reihenfolge in Zwillingsformeln wie drunter und drüber oder Hals über Kopf nicht gegen die Prinzipien der Ikonizität verstößt, da die Umkehrung der Reihenfolge als eine ikonische Abbildung der Unordnung interpretiert werden kann, die mithilfe dieser Phraseologismen ausgedrückt wird.

Neben den Arbeiten, die unmittelbar auf die Ikonizität von Zwillingsformeln Bezug nehmen, sind für die hier vorgenommene Analyse wissenschaftliche Positionen von Relevanz, in denen der Einfluss der Ikonizität auf die Reihenfolgebeziehungen in Wortpaaren ${ }^{9}$ thematisiert wird. Auch in diesen Positionen bildet die Ikonizität nur selten den zentralen Untersuchungsgegenstand. In den meisten Arbeiten aus der genannten Gruppe beschränkt sich die Beschreibung ikonischer Aspekte der linearen Anordnung von Bestandteilen der Wortpaare auf Kurzerwähnung solcher Erscheinungen wie die ikonische Abbildung der zeitlichen Abfolge von Ereignissen (cf. Benor/Levy 2006: 239-240; Lohmann 2012: 28; Mollin 2012: 87-88; Lohmann 2014: 33-34; Mollin 2014: 79; Renner 2014: 448), der Ursache-und-Wirkungsverhältnisse (cf. Benor/Levy 2006: 240; Mollin 2012: 87-88; Lohmann 2014: 34; Mollin 2014: 79) und anderer Skalen wie z. B. fortlaufende Nummerierung oder aufeinanderfolgende Bildungsstufen (cf. Lohmann 2012: 28; 2014: 34). Der Autorin des vorliegenden Textes sind ausschließlich vier Beiträge bekannt, in denen die Analyse der sequentiellen Ikonizität in Wortpaaren über die Angabe der genannten Tatsachen hinausgeht: Birdsong (1982), Nöth (1993), Birdsong (1995) und Boers/Lindstromberg (2008).

Birdsong (1982: 29) weist in seinen psycholinguistischen Untersuchungen zur Kindersprache, die u. a. Untersuchungsergebnisse zu Reihenfolgebeziehungen in Wortpaaren enthalten, darauf hin, dass die sequentielle Ikonizität in Zwillingsformeln neben der ikonischen Abbildung der zeitlichen Abfolge von Ereignissen und der Ursache-und-Wirkungsverhältnisse auch Fälle umfasst, in denen die Reihenfolgebeziehungen in einem Wortpaar die Bevorzugung des erst-

\footnotetext{
${ }^{9}$ Mit dem Terminus Wortpaar bezeichnen wir eine Verbindung von zwei Wörtern der gleichen Wortart, die sich durch denselben strukturellen Aufbau wie eine Zwillingsformel auszeichnet, in der aber die Reihenfolge der Bestandteile keine Merkmale der Festigkeit aufweist.
}

ISSN 1615-3014 
genannten Referenten durch das Kind bzw. die Nähe des Kindes zum erstgenannten Referenten widerspiegeln.

Birdsong (1995) untersucht den Einfluss der Ikonizität, der Markiertheit und der Sprachverarbeitungsfaktoren auf die Reihenfolge der von Kindern formulierten Wortpaare. Er kommt dabei zum Schluss, dass die in den Aussagen von Kindern zu beobachtenden Regelmäßigkeiten mit unterschiedlichen Arten von Ikonizität in Verbindung gebracht werden können. Erstens handle es sich bei der Anordnung von Elementen im Einklang mit der von Cooper/Ross (1975) formulierten Ich-vor-allem-Regel um eine ikonische Abbildung des für die Betrachtungsperspektive des Subjekts charakteristischen Vorrangs für den erstgenannten Referenten. Zweitens könne die im analysierten Korpus zu beobachtende Tendenz zur Voranstellung sprachlicher Einheiten, die auf zeitlich vorangehende Ereignisse bzw. Ursachen für später genannte Wirkungen Bezug nehmen, auf den ikonischen Charakter einer solchen Anordnung zurückgeführt werden. Drittens lasse sich anhand der durchgeführten Analyse feststellen, dass in den von Kindern formulierten Wortpaaren die semantische Markiertheit mit der Menge vom phonetischen Material korrespondiert, was in der Tat eine Art des phonetischen Symbolismus sei (cf. Birdsong 1995: 39-41). ${ }^{10}$

Nöth (1993: 26) vertritt die Ansicht, dass die Reihenfolge koordinierter sprachlicher Einheiten, darunter auch der Bestandteile von Wortpaaren, häufig als eine ikonische Abbildung verschiedener Typen von kognitiven, affektiven, sozialen und kulturellen Hierarchien betrachtet werden könne.

Boers/Lindstromberg (2008: 339) erwähnen drei sprachliche Erscheinungen in Wortpaaren, die mit der sequentiellen Ikonizität in Verbindung stehen:

- Die Reihenfolge sprachlicher Einheiten in einem Wortpaar spiegelt die zeitliche Abfolge von Ereignissen in der außersprachlichen Wirklichkeit wider.

- Die Reihenfolge sprachlicher Einheiten in einem Wortpaar ist eine ikonische Abbildung perzeptueller Markiertheit. Das Erstglied eines Wortpaars bezieht sich auf Referenten, die typischerweise früher wahrgenommen werden als Referenten, die das Zweitglied denotiert.

- Das erstgenannte Glied fungiert als Frame oder Hintergrund für das zweitgenannte.

Aus der oben angebrachten Beschreibung bisheriger Untersuchungen zur Ikonizität der Zwillingsformeln und Wortpaare wird ersichtlich, dass obwohl in der einschlägigen Literatur zu Zwillingsformeln die Analyse der linearen Anordnung ihrer Bestandteile zu zentralen Forschungsthemen gehört, der Einfluss der sequentiellen Ikonizität auf die Reihenfolgebeziehungen in Zwillingsformeln bisher nicht systematisch erforscht wurde. Dieses mangelnde Interesse an einer ausführlichen Analyse der Ikonizität in Zwillingsformeln kommt insbesondere darin zum Ausdruck, dass in der einschlägigen Literatur zu Zwillingsformeln und Wortpaaren die sequentielle Ikonizität nur im beschränkten Maße als ein unterschiedlichen Typen semantischer Hierarchien übergeordnetes allgemeines Prinzip betrachtet wird. Dem im vorliegenden

\footnotetext{
${ }^{10}$ Die letztgenannte Erscheinung betrifft auch unmittelbar die Reihenfolgebeziehungen in Wortpaaren, da sie auf eine Korrelation zwischen den semantischen und phonologischen Beschränkungen für die lineare Anordnung von Konstituenten Bezug nimmt.
} 
Beitrag formulierten Vorschlag, die sequentielle Ikonizität als ein allgemeines semantisches Prinzip in Betracht zu ziehen, steht die Arbeit von Nöth (1993) am nächsten, in der die Reihenfolgebeziehungen in Wortpaaren mit unterschiedlichen Modi sprachlicher Kodierung in Verbindung gebracht werden. Sie hat jedoch im Hinblick auf den Untersuchungsgegenstand des vorliegenden Artikels zwei Schwächen: Erstens lässt Nöth (1993) die Frage offen, welche semantischen Regeln mit den einzelnen Modi sprachlicher Kodierung korrespondieren. Zweitens ist für die Überprüfung der Relevanz von den bei Nöth (1993) formulierten Thesen für die Beschreibung deutscher Zwillingsformeln eine separate Studie erforderlich. Birdsong (1995) weist darauf hin, dass die von Cooper/Ross (1975) formulierte Ich-vor-allem-Regel den für die Betrachtungsperspektive des Subjekts charakteristischen Vorrang für den erstgenannten Referenten ikonisch widerspiegelt. Der genannte Vorschlag ist jedoch nur der erste Schritt zur Formulierung eines allgemeinen Prinzips, das die einzelnen semantischen Beschränkungen für die Reihenfolgebeziehungen in Zwillingsformeln mit sprachlicher Ikonizität in Verbindung brächte. Wie bereits Cooper/Ross (1975) bemerkt haben, können nämlich manche semantischen Beschränkungen für die lineare Anordnung von Bestandteilen der Zwillingsformeln nicht auf die Ich-vor-allem-Regel zurückgeführt werden. Auch die von Szpyra (1983) vorgeschlagene Erweiterung der Ich-vor-allem-Regel um Fälle, in denen die Reihenfolgebeziehungen in Zwillingsformeln die für die jeweilige Kultur charakteristische Wertehierarchie widerspiegeln, erlaubt nicht, alle semantischen Hierarchien auf ein einziges allgemeines Prinzip zurückzuführen. Ausnahmen bilden z. B. die Hierarchie ,göttlich - weltlich“ sowie Hierarchien, die auf unterschiedliche Skalen Bezug nehmen (z. B. die fortlaufende Nummerierung, aufeinanderfolgende Bildungsstufen), die nicht eindeutig als Fälle affektiver, kognitiver, sozialer bzw. kultureller Kodierung eingestuft werden können.

Im nächsten Teil des Beitrags überprüfen wir die Möglichkeit, die einzelnen semantischen Hierarchien, welche die Reihenfolgebeziehungen in Zwillingsformeln determinieren, mit unterschiedlichen Typen sequentieller Ikonizität in Verbindung zu bringen. Der Analyse liegt die These zugrunde, dass das Prinzip der sequentiellen Ikonizität als ein den einzelnen semantischen Regeln übergeordnetes allgemeines Prinzip zu betrachten ist, das verschiedene Modi sprachlicher Kodierung umfasst.

\section{$5 \quad$ Zum Einfluss der sequentiellen Ikonizität auf die lineare Abfolge der Bestandteile deutscher Zwillingsformeln}

In diesem Teil des Beitrags konzentrieren wir uns auf die Analyse der Relevanz vom Prinzip der sequentiellen Ikonizität für die Beschreibung der linearen Abfolge von Bestandteilen deutscher Zwillingsformeln. Mit dem Terminus sequentielle Ikonizität bezeichnen wir verschiedene Arten sprachlicher Kodierung, in denen die lineare Abfolge sprachlicher Einheiten die Struktur der außersprachlichen Wirklichkeit oder der kognitiven bzw. der soziokulturellen Kodierung ${ }^{11}$ widerspiegelt. Die vorliegende Analyse verfolgt zweierlei Zwecke. Erstens setz-

\footnotetext{
${ }^{11} \mathrm{Im}$ vorliegenden Beitrag vertreten wir die Ansicht, dass der Einfluss der soziokulturellen Kodierung auf die Reihenfolgebeziehungen in Zwillingsformeln nicht ausschließlich in Anlehnung an Daten zu kulturspezifischen Unterschieden zwischen Zwillingsformeln aus verschiedenen Sprachen analysiert werden kann, sondern auch in Anlehnung an Daten, die den soziokulturellen Wandel innerhalb einer Sprachgemeinschaft signalisieren (z. B. den Wandel der Geschlechterrollen), sowie Daten, deren Zusammenhang mit soziokulturellen Faktoren festgestellt werden kann, ohne dass ein Vergleich zwischen verschiedenen Kulturen erforderlich wäre.
} 
ten wir uns zum Zweck, die einzelnen semantischen und pragmatischen Faktoren auszusondern, welche die Reihenfolgebeziehungen in deutschen Zwillingsformeln determinieren. Zweitens wenden wir uns der Frage zu, inwieweit die Vielzahl von Faktoren, die mit dem Sammelbegriff Ikonizität bezeichnet werden, in einem Modell beschrieben werden kann, das die in diagrammatischen Ikons zu beobachtende Similaritätsbeziehung ausschließlich mit der Struktur der außersprachlichen Wirklichkeit, der kognitiven Kodierung bzw. der soziokulturellen Kodierung in Verbindung bringt. Das Korpus für die vorliegende Untersuchung bildet das Verzeichnis deutscher Zwillingsformeln, das der Monographie von Hans-Georg Müller Adleraug und Luchsenohr. Deutsche Zwillingsformeln und ihr Gebrauch entnommen wurde, und die Sammlung der gebräuchlichen Zwillingsformeln in der deutschen Gegenwartssprache von Wernfried Hofmeister. Die Analyse erfolgte in drei Etappen: In der ersten Etappe wurde anhand der ausgewählten Verzeichnisse eine Sammlung von Zwillingsformeln angefertigt, an der im nächsten Schritt eine Gliederung des Materials aufgrund der semantischen und pragmatischen Charakteristik der im Korpus vertretenen Zwillingsformeln vorgenommen wurde. Im letzten Schritt der Analyse waren wir bestrebt, die in der zweiten Etappe identifizierten semantischen und pragmatischen Faktoren, welche die Reihenfolge von Bestandteilen der deutschen Zwillingsformeln determinieren, auf verschiedene Typen sequentieller Ikonizität zurückzuführen, die mit der ikonischen Abbildung der Struktur der außersprachlichen Wirklichkeit, der kognitiven Kodierung bzw. der soziokulturellen Kodierung im Zusammenhang stehen.

In Anlehnung an die Daten aus dem analysierten Korpus kann festgestellt werden, dass die lineare Abfolge von Bestandteilen der Zwillingsformeln mit einer Vielzahl semantischer und pragmatischer Faktoren in Verbindung gebracht werden kann. Die erste Faktorengruppe ist mit der ikonischen Abbildung der Struktur der außersprachlichen Wirklichkeit verbunden. Es handelt sich in diesem Falle um Abfolgeprinzipien, die von der kognitiven und soziokulturellen Kodierung im hohen Grade unabhängig sind. ${ }^{12}$ Hierzu gehören Zwillingsformeln, in denen die zeitliche Abfolge von Ereignissen ikonisch abgebildet wird, sowie solche, in denen die Reihenfolgebeziehungen durch die Lebhaftigkeitshierarchie Mensch - Tier - Pflanze - unbelebte Materie determiniert werden:

\section{Zeitliche Abfolge}

Beispiele: damals wie heute, einst und jetzt, früher oder später, leben und sterben Ausnahmen: ${ }^{13}$ nach wie vor

\footnotetext{
12 Nach Malkiel (1959) und Jarosz (2009) können auch im Falle der hier angesprochenen Hierarchien bestimmte Aspekte der kognitiven und kulturellen Kodierung identifiziert werden (z. B. ergebe sich die Chronologie erst aus der Art und Weise, wie wir die Wirklichkeit wahrnehmen (cf. Jarosz 2009: 51); der in der Lebhaftigkeitshierarchie zu beobachtende Vorrang für Menschen sei ein Ausdruck der kulturellen Kodierung (cf. Malkiel 1959: 146)). Im vorliegenden Text vertreten wir jedoch die These, dass eine derartige Ablehnung des direkten Zusammenhangs zwischen der Struktur eines Ikons und der Struktur der außersprachlichen Wirklichkeit für die Interpretation der Daten aus dem Korpus nicht erforderlich ist.

${ }^{13}$ Im Hinblick auf den Untersuchungsgegenstand des vorliegenden Artikels werden bei der Besprechung der angebrachten Beispiele und Ausnahmen ausschließlich semantische Beschränkungen für die lineare Anordnung von Bestandteilen der Zwillingsformeln berücksichtigt. Wir schließen jedoch nicht die Möglichkeit aus, dass manche der während der Korpusanalyse identifizierten Ausnahmen auf phonologische Beschränkungen zurückgeführt werden können. Auffallend ist die hohe Anzahl von Ausnahmen, in denen die steigende Silbenzahl von Bestandteilen der Zwillingsformeln mit dem Gesetz der wachsenden Glieder im Einklang steht. Die statistische Relevanz der genannten Tendenz sowie - potentiell - anderer phonologischer Beschränkungen für die Beschreibung deutscher Zwillingsformeln müsste jedoch durch weitere Untersuchungen bestätigt werden.
} 


\title{
Mensch - Tier - Pflanze - unbelebte Materie
}

Beispiele: Herr und Hund, Mensch und Maschine, Pferd und Wagen, Bienchen und Blümchen Ausnahmen: z. B. Ross und Reiter, Waffen und Helden

Als Sonderfall der Lebhaftigkeitshierarchie können Zwillingsformeln betrachtet werden, in denen größere Tiere, häufig Raubtiere, die in der Nahrungskette kleineren Tieren übergeordnet sind, mit kleineren Tieren zusammengestellt werden (z. B. Katz' und Maus).

Die zweite Faktorengruppe umfasst Prinzipien, in denen die Reihenfolge von Bestandteilen einer Zwillingsformel biologisch und neuropsychologisch determinierte Tendenzen im Verlauf der Wahrnehmungsprozesse widerspiegelt. Beispiele für derartige Prinzipien sind die ikonische Darstellung des Grades an Aktivität sowie die Hierarchien oben - unten und großklein. In allen genannten Fällen ergibt sich die lineare Abfolge von Bestandteilen der Zwillingsformel aus Tendenzen, welche die visuelle Wahrnehmung kennzeichnen - die visuelle Wahrnehmung erfolgt typischerweise von oben nach unten (cf. Plank 1979: 140-141; Cooper/Klouda 1995: 337-338), die Aufmerksamkeit tendiert dazu, sich auf die aktiven bzw. größeren Teilnehmer der Szene zu fokussieren (cf. Churchland 1996: 238-239; Wogalter/Leonard 2005: 117):

\begin{abstract}
Aktiv - passiv
Beispiele: sehen und gesehen werden, tun oder lassen, fressen und gefressen werden, Geben und Nehmen
\end{abstract}

\section{Oben - unten}

Beispiele: auf und nieder, Berg und Tal, Hand und Fu $\beta$, rauf und runter

Ausnahmen: z. B. Rock und Bluse, Himmel und Erde/Erde und Himmel ${ }^{14}$

\section{Groß - klein}

Beispiele: weder Baum noch Strauch, Bomben und Granaten, groß und klein, Weg und Steg Ausnahmen: Busch und Baum, Kleines mit Großem

$\mathrm{Zu}$ Faktoren, die mit biologisch und neuropsychologisch bedingten Tendenzen im Verlauf der Wahrnehmungsprozesse in Verbindung stehen, gehört auch die Relevanz verschiedener sinnlicher Eindrücke. Hierzu gehören Zwillingsformeln, in denen Einheiten, die auf die visuelle Wahrnehmung Bezug nehmen, den Einheiten, die mit der akustischen Wahrnehmung verbunden sind, vorangehen:

\section{Visuelle vs. akustische Wahrnehmung}

Beispiele: Adleraug und Luchsenohr, Augen und Ohren, Bild und Ton, blind und taub Ausnahmen: z. B. Hören und Sehen, Rundfunk und Fernsehen

Die dritte Faktorengruppe bezieht sich auf die ikonische Darstellung von der Betrachtung der außersprachlichen Wirklichkeit aus der Perspektive des deiktischen Zentrums (der deiktischen Orientierung) bzw. hinsichtlich immanenter Eigenschaften bestimmter Objekte (der intrinsi-

\footnotetext{
14 Plank (1979: 41) und Mellado Blanco (1998: 292) vertreten die These, dass auch die Umkehrung der aus der Sicht der perzeptuellen Markiertheit bevorzugten Reihenfolge potentiell mit Ikonizität in Verbindung gebracht werden kann, da die Reihenfolge unten - oben in Zwillingsformeln wie drunter und drüber oder Hals über Kopf der ikonischen Abbildung der mithilfe dieser Zwillingsformeln ausgedrückten Unordnung dient.
} 
schen Orientierung). ${ }^{15}$ Mit dem Terminus deiktische Orientierung bezeichnen wir hier die Darstellung der Position einer Entität relativ zum deiktischen Zentrum, typischerweise dem Ego des Sprechers (z. B. die Verwendung des Satzes Das Fahrrad steht vor dem Haus zur Bezeichnung der Tatsache, dass sich das Fahrrad im Blickwinkel des deiktischen Zentrums, zwischen dem Haus und dem deiktischen Zentrum befindet) im Unterschied zur intrinsischen Orientierung, wo eine Entität in Bezug auf immanente Eigenschaften eines anderen Objekts lokalisiert wird (z. B. die Verwendung des Satzes Das Fahrrad steht vor dem Haus zur Bezeichnung der Tatsache, dass sich das Fahrrad vor der Eingangstür des Hauses befindet) (cf. Pörings/Schmitz 1999: 6-8). Die Tendenz, die Wirklichkeit aus der Perspektive des deiktischen Zentrums darzustellen, kommt in der Hierarchie nah - fern zum Ausdruck, die sich primär auf die räumlichen Verhältnisse bezieht und sekundär auf andere konzeptuelle Domänen übertragen werden kann. Dieser Gruppe von Faktoren kann auch die Hierarchie vorwärts - rückwärts zugeordnet werden, in der die Bewegungsrichtung in Relation zu immanenten Eigenschaften des Objekts bzw. zur Position des deiktischen Zentrums konzeptualisiert wird:

Nah - fern

Beispiele: dies und jenes, dieser und jener, hier und da, hüben und drüben

Ausnahmen: nah und fern/(von) fern und nah

Metaphorische Übertragung auf emotionale Nähe und Verwandtschaftsbeziehungen Beispiele: Familie und Freunde, weder verwandt noch verschwägert

\section{Vorwärts - rückwärts}

Beispiele: vor und zurück, vorwärts und rückwärts

Die vierte Faktorengruppe ${ }^{16}$ bezieht sich auf die Unterscheidung zwischen sinnlich wahrnehmbaren und abstrakten Entitäten. Die Hierarchie konkret - abstrakt, die mit der kognitiven Fähigkeit zur Kategorisierung im direkten Zusammenhang steht, dient zur ikonischen Darstellung der in der These über das Embodiment betonten Tatsache, dass die sinnliche Erfahrung im Vergleich zu anderen Typen von Erfahrungen bei der Strukturierung der Wirklichkeit die primäre Rolle spielt: ${ }^{17}$

\section{Konkret - abstrakt}

Beispiele: Luft und Liebe, Kopf und Verstand, Körper und Seele, Leib und Leben

Ausnahmen: z. B. Geist und Körper

\footnotetext{
15 Im vorliegenden Beitrag vertreten wir - im Unterschied zu Cooper/Ross (1975) und Ross (1980) - die Ansicht, dass unterschiedliche Typen semantischer Faktoren, die mit der Betrachtung der Welt aus der Sicht des prototypischen Sprechers in Verbindung stehen, voneinander abgegrenzt werden müssen: z. B. biologisch und neuropsychologisch bedingte Tendenzen im Verlauf der Wahrnehmungsprozesse, die Tendenz zur Strukturierung der Wirklichkeit entsprechend der Präferenzen des Konzeptualisierers, die Darstellung der Position einer Entität relativ zum deiktischen Zentrum, Faktoren, die auf die kognitive Fähigkeit zur Kategorisierung Bezug nehmen usw. Aufgrund der unzureichenden Evidenz für einen unmittelbaren Zusammenhang zwischen der Ichvor-allem-Regel und den sozial bzw. kulturell bedingten Hierarchien, der ikonischen Abbildung der zeitlichen Abfolge von Ereignissen sowie der Lebhaftigkeitshierarchie werden diese als separate Faktoren betrachtet, die von der Betrachtungsperspektive des Sprechers weitgehend unabhängig sind.

16 Aufgrund des besonderen Status des Unterschiedes zwischen der sinnlichen Wahrnehmung und kognitiven Prozessen, die sich auf abstrakte Entitäten beziehen, haben wir uns entschieden, die Hierarchie konkret - abstrakt einer separaten Faktorengruppe zuzuordnen.

${ }^{17}$ Für nähere Informationen zur These über das Embodiment s. z. B. Johnson (1987); Sinha/Jensen de López (2000).
} 
Die fünfte Faktorengruppe bezieht sich auf die ikonische Darstellung der subjektiven Erfahrung des Konzeptualisierers, der die Wirklichkeit entsprechend seiner Präferenzen, der Verteilung seiner Aufmerksamkeit bzw. der die kognitiven Prozesse der Schematisierung und Differenzierung kennzeichnenden Hierarchie konzeptueller Kategorien ${ }^{18}$ strukturiert. Hierzu gehören die Hierarchien positiv - negativ, neutral - negativ, prominent - weniger prominent, das Ganze - ein Teil des Ganzen und allgemein - spezifiziert:

\section{Positiv - negativ}

Beispiele: Chancen und Risiken, Freud und Leid, Lachen und Weinen, Zuckerbrot und Peitsche

Ausnahmen: z. B. Fluch oder Segen, Krieg und Frieden

\section{Prominent - weniger prominent}

Beispiele: Äpfel mit Birnen, Bett und Sofa, Punkt und Beistrich, Tür und Tor

\section{Allgemein - spezifiziert}

Beispiele: Mensch und Christ, Obst und Südfrüchte

Ausnahmen: Recht und Gesetz/Gesetz und Recht

\section{Das Ganze - ein Teil des Ganzen}

Beispiele: Bund und Länder, Kapitel und Vers, Zeit und Stunde

Die sechste Faktorengruppe betrifft Prinzipien, in denen die lineare Abfolge sprachlicher Einheiten bestimmte Aspekte der soziokulturellen Kodierung widerspiegelt. Hierzu gehört die ikonische Abbildung verschiedener Typen sozialer Hierarchien (der Unterordnung, des sozialen Status von Vertretern verschiedener Berufe, der Unterschiede im sozialen Status, die sich aus der Herkunft bzw. dem Alter ergeben; des sozialen Status der beiden Geschlechter in der patriarchalischen Gesellschaftsstruktur), die ikonische Abbildung der gesellschaftlich normierten Wertskala sowie der Unterschiede zwischen mündlicher und schriftlicher Kommunikation. Der Einfluss der soziokulturellen Kodierung ist auch in neu entstandenen Zwillingsformeln sichtbar, in denen die Voranstellung der sprachlichen Einheiten, die auf Personen weiblichen Geschlechts Bezug nehmen, als Ausdruck der Höflichkeit betrachtet wird und eine Abkehr von der patriarchalischen Gesellschaftsstruktur signalisiert:

\section{Sozialer Status}

Beispiele: Herr und Knecht (Unterordnung), Arzt oder Apotheker (sozialer Status der Vertreter verschiedener Berufe), Bürger und Bauern (Herkunft), Neffen und Nichten (patriarchalische Gesellschaftsstruktur), Vater und Sohn (Alter)

\section{Höflichkeit}

Beispiel: Damen und Herren

\footnotetext{
18 Mit dem Terminus Schematisierung, der für den Zweck der vorliegenden Analyse der Kognitiven Grammatik entnommen wurde, bezeichnen wir die mit der kognitiven Fähigkeit zur Abstrahierung verbundene Unterscheidung zwischen dem Schema und seinen Instanzen (cf. Tabakowska 2001: 57-58). Die ikonische Darstellung des kognitiven Prozesses der Schematisierung ist in denjenigen sprachlichen Strukturen zu beobachten, in denen die übergeordnete Kategorie (das Schema) der untergeordneten Kategorie (der Instanz) vorangeht (z. B. die Hierarchie allgemein - spezifiziert). Mit dem Terminus Differenzierung bezeichnen wir die kognitive Fähigkeit zur Unterscheidung von strukturellen Bestandteilen einer komplexen Einheit. Die ikonische Abbildung des kognitiven Prozesses der Differenzierung kommt in der Hierarchie das Ganze - ein Teil des Ganzen zum Ausdruck.
} 


\section{Wertskala}

Beispiele: Gold und Silber, Kunst und Gewerbe, Stadt und Land, Ostern und Pfingsten Ausnahmen: Teller und Silber, Stahl und Eisen/Eisen und Stahl

\section{Mündlich - schriftlich}

Beispiele: (in) Rede und Schrift, (in) Wort und Schrift

Die siebte Faktorengruppe bilden Prinzipien, in denen die lineare Abfolge sprachlicher Einheiten logische Zusammenhänge (Ursache - Wirkung, Mittel - Zweck, Umstand - erwarteter Begleitumstand) widerspiegelt:

\section{Ursache - Wirkung}

Beispiele: Forschung und Entwicklung, Sieg und Ruhm

\section{Mittel - Zweck}

Beispiele: Mittel und Zweck, suchen und finden, säen und ernten

\section{Umstand - erwarteter Begleitumstand}

Beispiele: alt und grau, alt und weise, jung und munter, satt und zufrieden

Darüber hinaus wurden im analysierten Korpus Tendenzen in der linearen Anordnung von Bestandteilen der Zwillingsformeln identifiziert, die keiner der oben genannten Faktorengruppen eindeutig zugeordnet werden können. Hierzu gehören die folgenden Hierarchien: bejaht - verneint, sacrum - profanum, fest - flüssig, hell - dunkel, warm - kalt, Anfang - Ende (hier sei auf den Zusammenhang zwischen räumlicher Orientierung, zeitlicher Abfolge und kulturell bedingten Abfolgeketten hingewiesen) und die Ikonizität zum Ausdruck der Steigerung ${ }^{19}$ :

\section{Anfang - Ende}

Beispiele: Alpha und Omega, Anfang und Ende

\section{Bejaht - verneint}

Beispiele: alles und nichts, Fug und Unfug, jetzt oder nie, überall und nirgends

\section{Steigerung}

Beispiele: Angst und Schrecken, kalt und frostig, Wissen und Weisheit, Wut und Hass

\section{Sacrum - profanum}

Beispiele: Gott und König, Gott und die Welt

\section{Fest - flüssig}

Beispiele: Essen und Trinken, Hunger und Durst, Land und Meer, Leib und Blut

Ausnahmen: z. B. Kaffee und Kuchen, Wasser und Brot

\section{Hell - dunkel}

Beispiele: Licht und Finsternis, Licht und Schatten, Tag und Nacht

\footnotetext{
${ }^{19}$ Da den Untersuchungsgegenstand des vorliegenden Artikels die sequentielle Ikonizität in deutschen Zwillingsformeln darstellt, verwenden wir hier den Terminus Ikonizität zum Ausdruck der Steigerung in Bezug auf die ikonische Abbildung der Intensität von Bestandteilen der Zwillingsformeln durch Voranstellung des Elements, dessen Bedeutung sich durch einen niedrigeren Grad an Intensität auszeichnet. Es sei jedoch betont, dass die angebrachten Beispiele auch die Verwendung der quantitativen Ikonizität zum Ausdruck der Steigerung illustrieren, da die Wiederholung desselben semantischen Gehalts durch Synonyme ikonisch auf die bedeutungsintensivierende Funktion der Zwillingsformeln verweist.
} 


\section{Warm - kalt}

Beispiele: heiß und kalt, Hitze und Kälte, Sommer und Winter

Ausnahmen: kalt und warm

\section{$6 \quad$ Zusammenfassung}

Zusammenfassend können wir feststellen, dass die lineare Abfolge von Bestandteilen der Zwillingsformeln durch zahlreiche Faktoren determiniert ist, die mit dem Prinzip der sequentiellen Ikonizität in Verbindung gebracht werden können. Hierzu gehören insbesondere die folgenden drei Gruppen von Faktoren:

- Faktoren, in denen die strukturelle Abbildung der außersprachlichen Wirklichkeit von der kognitiven und soziokulturellen Kodierung im hohen Grade unabhängig ist

- Faktoren, die auf verschiedene Typen kognitiver Prozesse Bezug nehmen (z. B. die biologisch und neuropsychologisch determinierten Präferenzen im Verlauf der Wahrnehmungsprozesse, die Betrachtung der Wirklichkeit aus verschiedenen Perspektiven im Einklang mit der deiktischen und der intrinsischen Orientierung, den nach der These über das Embodiment primären Unterschied zwischen sinnlicher Erfahrung und anderen Typen von Erfahrungen, die Fähigkeit zur Erkennung logischer Zusammenhänge oder die subjektive Erfahrung des Konzeptualisierers, der die Wirklichkeit entsprechend seiner Präferenzen, der Verteilung seiner Aufmerksamkeit bzw. der die kognitiven Prozesse der Schematisierung und Differenzierung kennzeichnenden Hierarchie konzeptueller Kategorien strukturiert)

- Faktoren, die mit bestimmten Aspekten der soziokulturellen Kodierung in Verbindung stehen.

Darüber hinaus wurden im analysierten Korpus einige Tendenzen in der linearen Anordnung von Bestandteilen der Zwillingsformeln identifiziert, die keiner der genannten Faktorengruppen eindeutig zugeordnet werden können. Die Vielzahl von Tendenzen, die mit dem Sammelbegriff sequentielle Ikonizität bezeichnet werden, zeugt davon, dass eine detaillierte Beschreibung der sprachlichen Ikonizität ausschließlich in einem Modell möglich ist, in dem außer der unmittelbaren Beziehung zwischen dem ikonischen Zeichen und der außersprachlichen Wirklichkeit auch verschiedene Aspekte der kognitiven und soziokulturellen Kodierung berücksichtigt werden.

\section{Korpus}

Hofmeister, Wernfried (2010): „Sammlung der gebräuchlichen Zwillingsformeln in der deutschen Gegenwartssprache“. http://zwillingsformeln.unigraz.at/ZWILLINGSFORMELN\% 20Hofmeister\%2025-06-2010.pdf [06.10.2014].

Müller, Hans-Georg (2009): Adleraug und Luchsenohr. Deutsche Zwillingsformeln und ihr Gebrauch. Frankfurt a. M.: Lang.

\section{Literatur}

Andersen, Earl R. (1998): A Grammar of Iconism. Madison, New Jersey: Fairleigh Dickinson University Press. 
Behaghel, Otto (1909): „Beziehungen zwischen Umfang und Reihenfolge von Satzgliedern“. Indogermanische Forschungen 25: 110-142.

Behaghel, Otto (1932): Deutsche Syntax. Eine geschichtliche Darstellung. Bd. 4: Wortstellung. Periodenbau. Heidelberg: Winters.

Benor, Sarah Bunin/Levy, Robert (2006): "The chicken or the egg? A probabilistic analysis of English binomials". Language 82/2: 233-277.

Birdsong, David (1982): "Semantics of word order in co-ordination". Papers and Reports on Child Language Development 21: 25-32.

Birdsong, David (1995): "Iconicity, markedness, and processing constraints in frozen locutions”. In: Landsberg, Marge E. (ed.): Syntactic Iconicity and Linguistic Freezes. Berlin, de Gruyter: 31-45.

Boers, Frank/Lindstromberg, Seth (2008): "Structural elaboration by the sound (and feel) of it". In: Boers, Frank/Lindstromberg, Seth (eds.): Cognitive Linguistic Approaches to Teaching Vocabulary and Phraseology. Berlin, de Gruyter: 329-354.

Bolinger, Dwight L. (1962): "Binomials and pitch accent". Lingua 11: 34-44.

Burger, Harald (2010): Phraseologie: eine Einführung am Beispiel des Deutschen. Berlin: Schmidt.

Churchland, Paul (1996): The engine of reason, the seat of the soul: a philosophical journey into the brain. Cambridge, Mass./London: MIT Press.

Cooper, William E./Klouda, Gayle V. (1995): "The psychological basis of syntactic iconicity". In: Landsberg, Marge E. (ed.): Syntactic Iconicity and Linguistic Freezes. Berlin, de Gruyter: 331-342.

Cooper, William E./Ross, John R. (1975): "World Order”. In: Grossmann, Robin E./San, L. James/Vance, Timothy J. (eds.): Papers from the Parasession on Functionalism. Chicago, University of Chicago Press: 63-111.

Croft, William (1995): “Modern Syntactic Typology”. In: Shibatani, Masayoshi et al. (eds.): Approaches to Language Typology: Past and Present. Oxford, Oxford University Press: 85-144.

Dotter, Franz (1995): "Nonarbitrariness and Iconicity: Coding Possibilities". In: Landsberg, Marge E. (ed.): Syntactic Iconicity and Linguistic Freezes. Berlin, de Gruyter: 47-55.

Farø, Ken (2006a): Idiomatizität - Ikonizität - Arbitrarität: Beitrag zu einer funktionalistischen Theorie der Idiomäquivalenz. Kopenhagen: Museum Tusculanum. http://curis.ku.dk/ws/files/38379427/Ph.d._afhandling_Ken_Far_2006.pdf [03.06.2016].

Farø, Ken (2006b): „Ikonographie, Ikonizität und Ikonizismus: Drei Begriffe und ihre Bedeutung für die Phraseologieforschung“. Linguistik online, 27/2: 57-71. http://www.linguistikonline.org/27_06/faroe.pdf [03.06.2016].

Fenk-Oczlon, Gertraud (1989): "Word frequency and word order in freezes". Linguistics 27: 517-556.

Givón, Talmy (1989): Mind, Code and Context. Essays in Pragmatics. Hillsdale/NJ: Erlbaum.

Givón, Talmy (1995): "Isomorphism in the grammatical code: cognitive and biological considerations”. In: Simone, Raffaele (ed.): Iconicity in Language. Amsterdam, John Benjamins: 47-76.

Haiman, John (1985): Natural Syntax. Iconicity and Erosion. Cambridge: Cambridge University Press. 
Haspelmath, Martin (2003): “Against Iconicity and Markedness". Max-Planck-Institut für evolutionäre Anthropologie in Leipzig. http:/www.eva.mpg.de/lingua/staff/haspelmath/ pdf/IconicityMarkedness.pdf [15.06.2014].

Hüpper, Dagmar/Topalović, Elvira/Elspaß, Stephan (2002): „Zur Entstehung und Entwicklung von Paarformeln im Deutschen“. In: Piirainen, Elisabeth/Piirainen, Ilpo Tapani (eds.): Phraseologie in Raum und Zeit. Akten der 10. Tagung des Westfälischen Arbeitskreises „Phraseologie/Parömiologie“ (Münster 2001). Hohengehren, Schneider: 77-99.

Jarosz, Józef (2009): „Deutsche und dänische Zwillingsformeln im Vergleich“. In: Bartoszewicz, Iwona et al. (eds.): Germanistische Linguistik extra muros - Aufgaben. Wrocław, Oficyna Wydawnicza ATUT: 47-56.

Johnson, Mark (1987): The Body in the Mind: the bodily basis of meaning, imagination and reason. Chicago: University of Chicago Press.

Kimenyi, Alexandre (1989): "Reduplication and binomial expressions in English: A case of syntagmatic iconicity”. In: Rauch, Irmengard/Carr, Gerald F. (eds.): The Semiotic Bridge. Trends from California. Berlin, de Gruyter: 347-353.

Lohmann, Arne (2012): "A processing view on order in reversible and irreversible binomials”. Vienna English Working Papers, 21: 25-50. https://anglistik.univie.ac.at/fileadmin/ user_upload/dep_anglist/weitere_Uploads/Views/VIEWS_21_2012_Lohmann.pdf [03. 06. 2016].

Lohmann, Arne (2014): English Coordinate Constructions. Cambridge: Cambridge University Press.

Malkiel, Yakov (1959): "Studies in Irreversible Binomials”. Lingua 8: 113-160.

Mellado Blanco, Carmen (1998): „Historische Entwicklung der deutschen Paarformeln mit somatischen Komponenten“. Neuphilologische Mitteilungen, 99/3: 285-295.

Mollin, Sandra (2012): "Revisiting binomial order in English: Ordering constraints and reversibility”. English Language and Linguistics 16/1: 81-103.

Mollin, Sandra (2014): The (Ir)reversibility of English Binomials: Corpus, constraints, developments. Amsterdam: John Benjamins.

Morris, Charles (1938/1964): Signification and Significance: A Study of the Relation of Signs and Values. Cambridge, Mass.: MIT Press.

Müller, Gereon (1997): „Beschränkungen für Binomialbildung im Deutschen. Ein Beitrag zur Interaktion von Phraseologie und Grammatik“. Zeitschrift für Sprachwissenschaft, 16: 551 .

Müller, Hans-Georg (2009): Adleraug und Luchsenohr. Deutsche Zwillingsformeln und ihr Gebrauch. Frankfurt a. M.: Lang.

Munat, Judith (2005): "Iconic functions of phraseological units and metaphor". In: Maeder, Costantino/Fischer, Olga/Herlofsky, William J. (eds.): Outside-In - Inside-Out. Amsterdam, Benjamins: 389-410.

Nöth, Winfried (1993): "Iconicity of symmetries and asymmetries in syntactic coordination". In: Küper, Christoph (ed.): Von der Sprache zur Literatur. Motiviertheit im sprachlichen und im poetischen Kode. Tübingen, Stauffenburg: 23-36.

Peirce, Charles Sanders (1903/1998): “A Syllabus of Certain Topics of Logic”. In: Peirce, Charles Sanders: The Essential Peirce. Selected Philosophical Writings. Bd. 2. Bloomington, Indiana University Press, Bd. 2: 258-299. 
Plank, Frans (1979): „Ikonisierung und De-Ikonisierung als Prinzipien des Sprachwandels“. Sprachwissenschaft 4/2: 121-158.

Pörings, Ralf/Schmitz, Ulrich (eds.) (1999): Sprache und Sprachwissenschaft. Eine kognitiv orientierte Einführung. Tübingen: Narr.

Renner, Vincent (2014): “A Study of Element Ordering in English Coordinate Lexical Items”. English Studies 95/4: 441-458. https://hal.archives-ouvertes.fr/hal-01097876/document [03.06.2016].

Römer, Christine (2007): „Ikonische Kodierung bei der Phraseologisierung von Verbphrasen“. In: Gallmann, Peter/Lehmann, Christian/Lühr, Rosemarie (eds.): Sprachliche Motivation - zur Interdependenz von Inhalt und Ausdruck. Tübingen, Narr: 203-218.

Ross, John Robert (1980): „Ikonismus in der Phraseologie. Der Ton macht die Bedeutung“. Zeitschrift für Semiotik 2: 39-56.

Schröter, Ulrich (1980): „Paarformeln in Gegenwart und Geschichte der deutschen Sprache“. Sprachpflege 29/10: 193-195.

Sinha, Chris/Jensen de López, Kristine (2000): "Language, Culture and the Embodiment of Spatial Cognition". Cognitive Linguistics 11: 17-41.

Sobkowiak, Włodzimierz (1993): "Unmarked-before-marked as a freezing principle”. Language and Speech 36/4: 393-414.

Southern, Mark Roderick Vendrell (2000): "Formulaic binomials, morphosymbolism, and Behaghel's Law: The grammatical status of expressive iconicity". American Journal of Germanic Linguistics and Literatures 12/2: 251-279.

Szpyra, Jolanta (1983): "Semantic and phonological constraints on conjunct ordering in English and Polish". Kwartalnik neofilologiczny 30: 33-53.

Tabakowska, Elżbieta (2001): Językoznawstwo kognitywne a poetyka przekładu. Kraków: Universitas.

Tabakowska, Elżbieta (2005): "Iconicity as a function of point of view”. In: Maeder, Costantino/Fischer, Olga/Herlofsky, William J. (eds.): Outside-In - Inside-Out. Amsterdam, Benjamins: 375-387.

Wescott, Roger W. (1971): "Linguistic Iconism”. Language 47: 417-428.

Wogalter, Michael S./Leonard, David S. (2005): “Attention Capture and Maintenance". In: Wogalter, Michael S./DeJoy, David M./Laughery, Kenneth R. (eds.): Warnings and Risk Communication. London, Taylor \& Francis: 113-138. 